\title{
TUNABLE FREQUENCY SURFACE DESIGN BETWEEN 2.43GHZ AND 6GHZ
}

\author{
Bora Döken $^{1}$ and Mesut Kartal ${ }^{2}$ \\ ${ }^{1}$ Department of Electronics and Communications Engineering, Istanbul Technical \\ University, Istanbul, Turkey \\ ${ }^{2}$ Department of Electronics and Communications Engineering, Istanbul Technical \\ University, Istanbul, Turkey
}

\begin{abstract}
Reconfigurable frequency selective surfaces (FSSs) which have more than one frequency response are demanded by recent communication systems. Tuneable FSS design is presented as a solution proposal to these demands in this work. Four-legged loaded element geometry is modified in order to achieve wide tuning range by inclusion of varactor diodes. Frequency tuning range is increased \%11 by comparing with the "Four Legged Loaded" element geometry. Achieved results show that proposed structure allows tuning between $2.42 \mathrm{GHz}-5.94 \mathrm{GHz}$ frequency bands. Analyses are executed with Ansoft HFSS v.15 software.
\end{abstract}

\section{KEYWORDS}

Frequency selective surface, FSS, periodic structures, active FSS, reconfigurable FSS, varactor diodes

\section{INTRODUCTION}

Periodic conducting structures exhibit different filter characteristics when interacting with incoming electromagnetic waves[1,2]. These periodic structures are called as frequency selective surfaces (FSSs). They can be divided into two main categories: reconfigurable FSSs or nonreconfigurable FSSs. Non-configurable FSSs generally have one frequency response[1-6]. Reconfigurable FSSs which have more than one frequency response are intensively researched nowadays[7-18]. Reconfigurable FSSs are demanded due to the intensive usage of radio propagation in indoor environment. Mutual interference between adjacent wireless networks in indoor environment is becoming an important issue due to reducing the communication speed significantly[19-25]. Isolating the wireless networks by using frequency selective surfaces (FSSs) can be an efficient solution for such interference problems. Non-configurable FSSs cannot be an effective solution due to the changing environment conditions. Frequency spectrum is needed to be utilized effectively especially in indoor environments. Indoor environment radio communication protocols are defined between $300 \mathrm{MHz}$ and $6 \mathrm{GHz}$ frequencies[17]. Therefore, a new band stop FSS geometry which can be tuned in wireless frequencies of indoor environment (between $2.42 \mathrm{GHz}$ and $3.94 \mathrm{GHz}$ ) is proposed in this work.

Reconfigurable frequency selective feature is mostly acquired by the inclusion of lumped elements, such as varicaps, PIN diodes and etc. in specific locations within each unit cell[17, 26, 27]. Inclusion of lumped elements allows control on the frequency response by changing the applied bias voltage. Reconfigurable FSSs can be divided into two main categories: switchable[15, 27] and tunable $[8,9,12,17,18,28,29]$. Tuning feature allows adjusting the resonant frequency response to the desired frequency bands. Besides, fabrication and installation

DOI: 10.14810/elelij.2017.6301 
errors can also be compensated. This feature is mostly acquired by inclusion of varactor diodes into specific locations in unit cell. Number and locations of the varactor diodes in unit cell, their electrical properties and FSS geometry mainly affect the tuning performance. Varactor diodes represent different equivalent capacitance values depending on the applied reverse biased voltage. Therefore, resonant frequencies of FSSs can be shifted by adjusting the applied voltages to active components.

In this work, "Four Legged Loaded"[1]is chosen as initial unit cell geometry (FSS). Unit cell geometries are connected to each other by inclusion of varactor diodes at the end of the legs where induced charges condense. Therefore, unit cell geometries itself are used to bias varactor diodes as a biasing grid. Wide tuning performance is achieved by placing varactor diodes where induced charges condenses. Subsequently, in order to achieve wider tuning range, four legged loaded geometry is modified by widening the end of the legs. Analyzes and optimization of the proposed surface were executed with Ansoft HFSS v.15 software which uses numerical techniques. Besides, equivalent circuit model were used to define the unit cell behavior of FSS at the design and optimization stages. Achieved results show that wide tuning range between $2.42 \mathrm{GHz}$ and $5.94 \mathrm{GHz}$ frequencies is achieved by using a simple structure on one layer.

In this paper, design process, simulation and measurement results are presented in Section II and the results are discussed in Section III.

\section{DeSign \& Measurement}

In this design, "Four Legged Loaded" is chosen as initial unit cell (FSS) geometry due to having stable frequency response and simple in structure. "Four Legged Loaded" FSS and its equivalent circuitare shown in Figure 1.Equivalent circuit model (EC) is used to define the behavior of FSS at the design and optimization stages. According to EC model, the equivalent capacitance value $\left(C \propto \frac{s}{g}\right)$ is mainly determined by the gap distance $(\mathrm{g})$ and the width of the gap (s) between periodic element geometries. Equivalent inductance value $\left(L \propto \frac{d}{w}\right)$ is mainly determined by the length (d) and the width $(\mathrm{w})$ of the current path on the periodic element geometries. The equivalent impedance and the resonant frequency equations (Eq. 1) of the FSS are derived from the given equivalent circuit model.

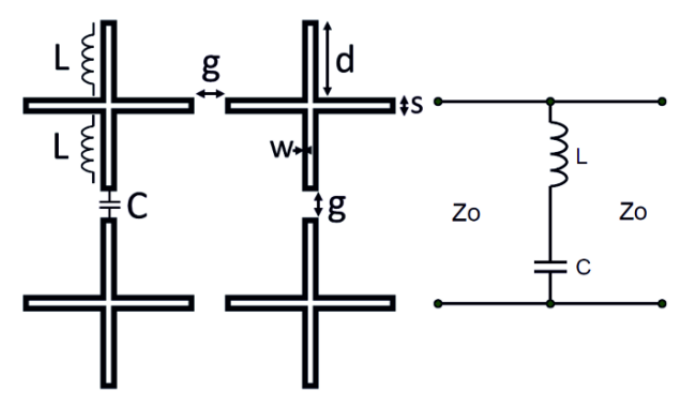

Figure 1. "Four Legged Loaded" FSS and its equivalent circuit

$$
Z=\frac{1-w^{2} L C}{j w C} f_{\mathrm{r}}=\frac{1}{2 \pi \sqrt{L C}}
$$

It is obvious from Eq. 1 that the resonant frequency of FSS can be tuned by changing the equivalent capacitance value $(C)$. Varactor diodes behave as a variable capacitor $\left(\mathrm{C}_{\mathrm{p}}\right)$ depending on the applied bias voltage. Therefore, in this work, varactor diodes are placed between unit cell 
geometries in order to adjust equivalent capacitance value $\left(C, C=C_{g}+C_{p}\right)$. " $C_{g}$ " and " $C_{p}$ " denotes the equivalent capacitance between geometries and equivalent capacitance of varactor diodes respectively. The SMV2201-040LF varactor diode is chosen in the design. Its equivalent capacitance values are between $0.23 \mathrm{pF}-2.1 \mathrm{pF}$ depending on the applied reverse bias voltage. Resistances $(2 \mathrm{k} \Omega)$ are placed parallel to the varactor diodes in order to reverse bias them. $2 \mathrm{k} \Omega$ resistance value is chosen considering the minimum impedance value of the varactor diodes and to limit the flowing current value. Analyzes of the proposed periodic structure are executed with Ansoft HFSS v.15 software which is based on finite element method. Proposed FSS (Figure 1) is optimized according to relationships explained above. Figure 2-3 show obtained simulation results for different capacitance values of varactor diodes for 30 degrees of incidence angles $(\theta)$. According to the achieved results, minimum $30 \mathrm{~dB}$ attenuation is obtained in the desired frequency band between $5.78 \mathrm{GHz}$ and $2.62 \mathrm{GHz}$ frequencies at TE polarization. However, lower quality factor $(\mathrm{Q})$ of the LC circuit widens "-20dB" bandwidth for large values of varactor capacitance. Besides, resistors of the biasing circuit and the dielectric material employed increase the transmission losses outside the resonant frequency bands.

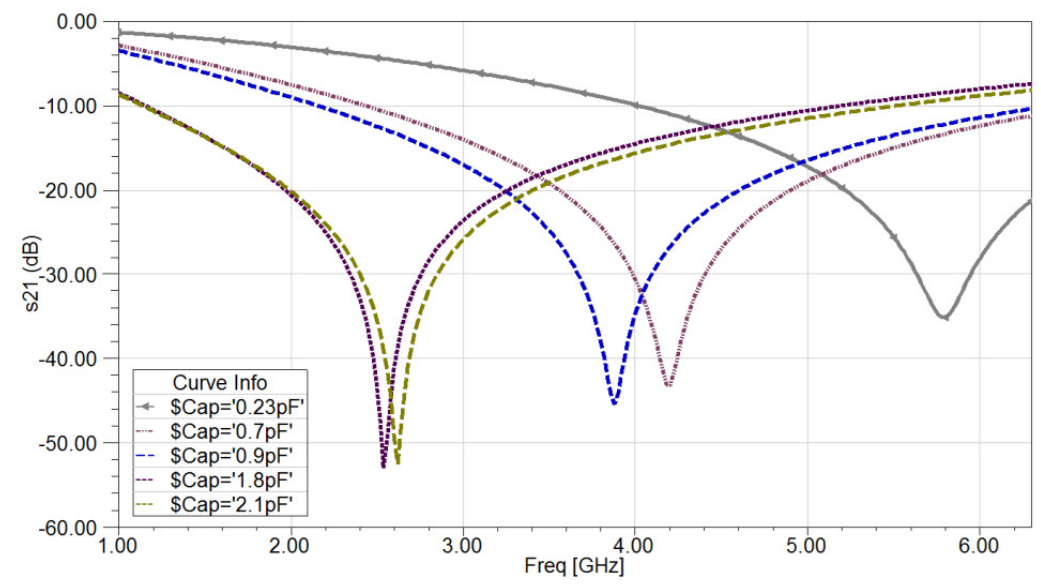

Figure 2. $S_{21}$ curves for different equivalent capacitance values (TE polarization, $\theta=30^{\circ}$ )

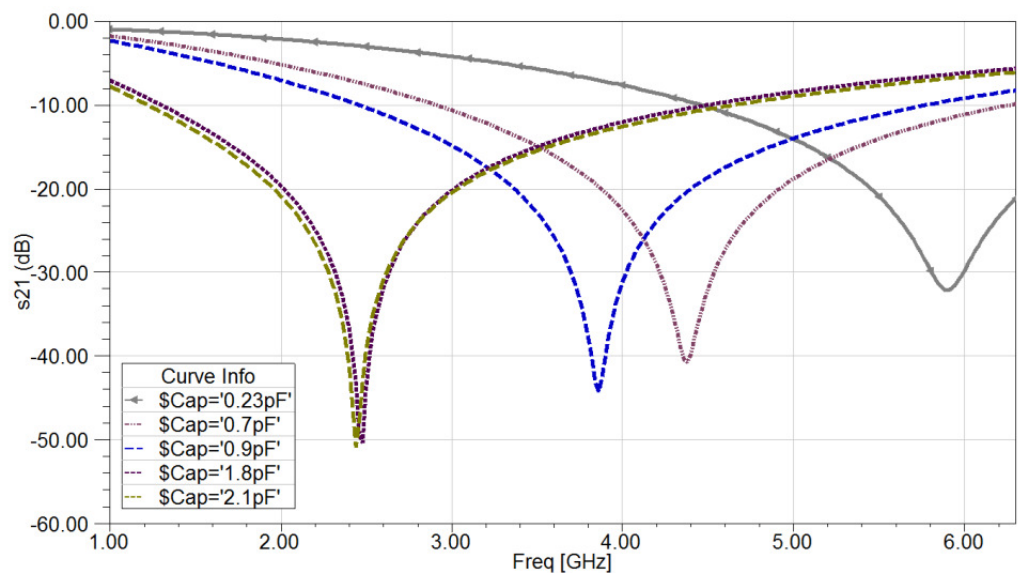

Figure 3. $S_{21}$ curves for different equivalent capacitance values (TM polarization, $\theta=30^{0}$ )

Subsequently, in order to achieve wider tuning range, four legged loaded geometry is modified by increasing (parameter $\mathrm{c}$ ) the end widths of the legs as shown in Figure 4. All the other parameters (except c) are kept same. Analyzes of the proposed periodic structure are executed with Ansoft 
Electrical and Electronics Engineering: An International Journal (ELELIJ) Vol.6, No.3, August 2017

HFSS v.15. Proposed FSS (Figure 3) is optimized according to relationships explained above. The following dimensions are obtained at the end of the optimization process: $h=0.8 \mathrm{~mm}$ (thickness of the FR4 substrate) and the other values in $(\mathrm{mm})$ are $\mathrm{p}=6.4, \mathrm{a}=1, \mathrm{~b}=2.35, \mathrm{c}=0.4$, $\mathrm{w}=0.2, \mathrm{pl}=0.3$.

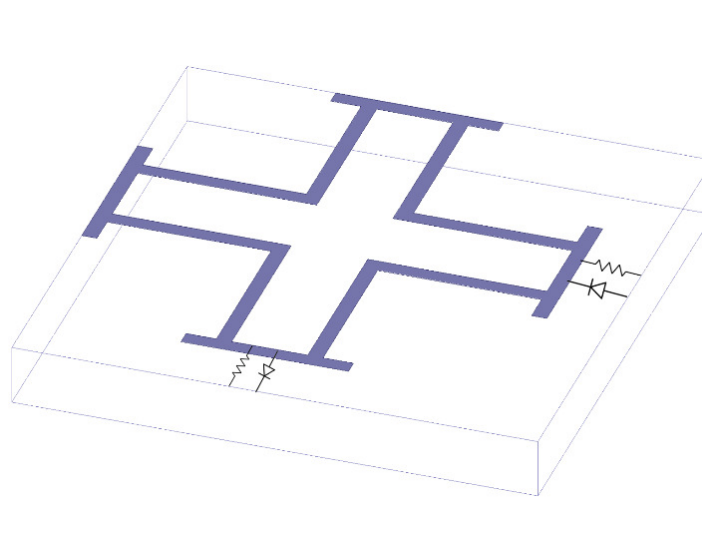

(a)

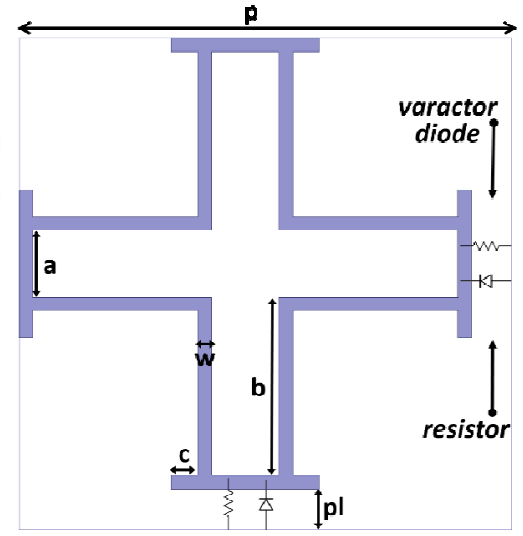

(b)

Figure 3. FSS geometry (a) Trimetric view (b) Top view

Figure 4-5 show obtained simulation results for different capacitance values of varactor diodes when the angle of incidence $(\theta)$ is 30 degrees. According to the achieved results, minimum $30 \mathrm{~dB}$ attenuation is obtained in the desired frequency band between $5.94 \mathrm{GHz}$ and $2.42 \mathrm{GHz}$ frequencies at TE polarization. Tuning range is increased \%11 comparing with the "Four Legged Loaded" element geometry. However, resonant bandwidths increases due to the increased equivalent capacitance $\left(\mathrm{C}_{\mathrm{g}}\right)$ values between unit cell geometries.

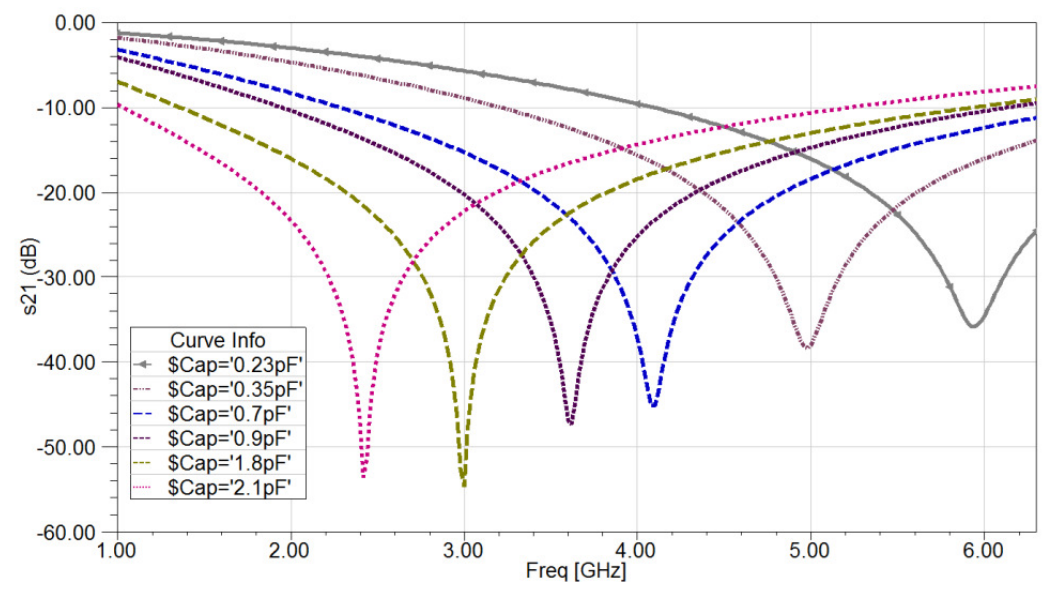

Figure 4. $S_{21}$ curves for different equivalent capacitance values (TE polarization, $\theta=30^{0}$ ) 
Electrical and Electronics Engineering: An International Journal (ELELIJ) Vol.6, No.3, August 2017

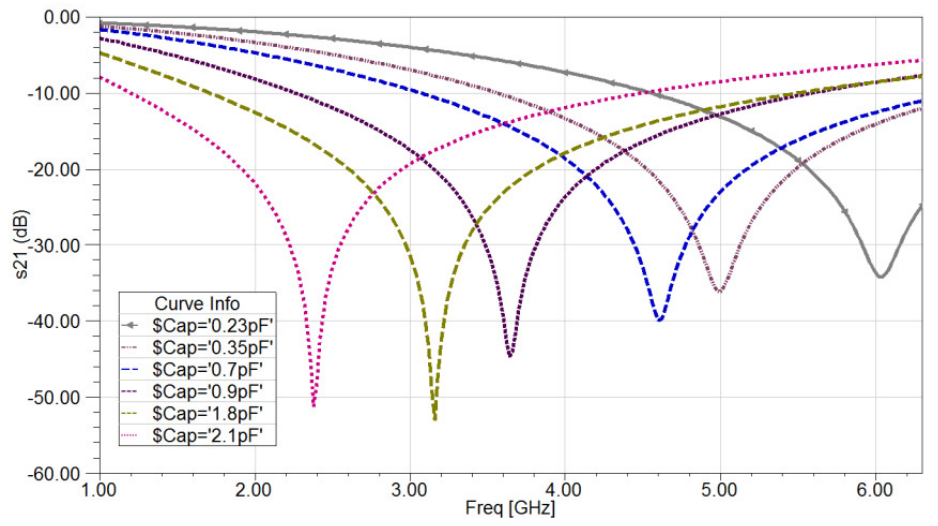

Figure 5. $\mathrm{S}_{21}$ curves for different equivalent capacitance values (TM polarization, $\theta=30^{0}$ )

Figure 6-7 show the simulation results for limit equivalent capacitance values $(0.35 \mathrm{pF}, 1.8 \mathrm{pF})$ at $0^{\circ}, 15^{\circ}$ and $30^{\circ}$ of incidence angles for both TE and TM polarizations respectively. Achieved results show that minimum $30 \mathrm{dBattenuation}$ is obtained in the desired frequency band for the oblique incidence angle ranging from normal to $30^{\circ}$. However, observed frequency shift relative to normal incidence is about $10 \%$ at TE30 and 4\% at TM30.Measurement results show that the incorporation of the varactor diodes degrade the angular stability, as expected [16].

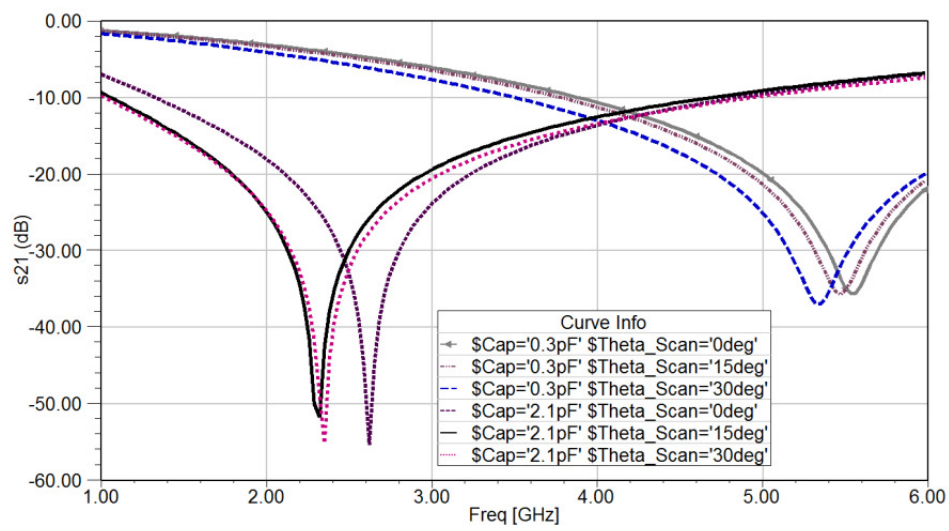

Figure 6. $S_{21}$ curves for different incidence angles (TE polarization, $\theta=0^{0}, \theta=15^{0}, \theta=30^{\circ}$ )

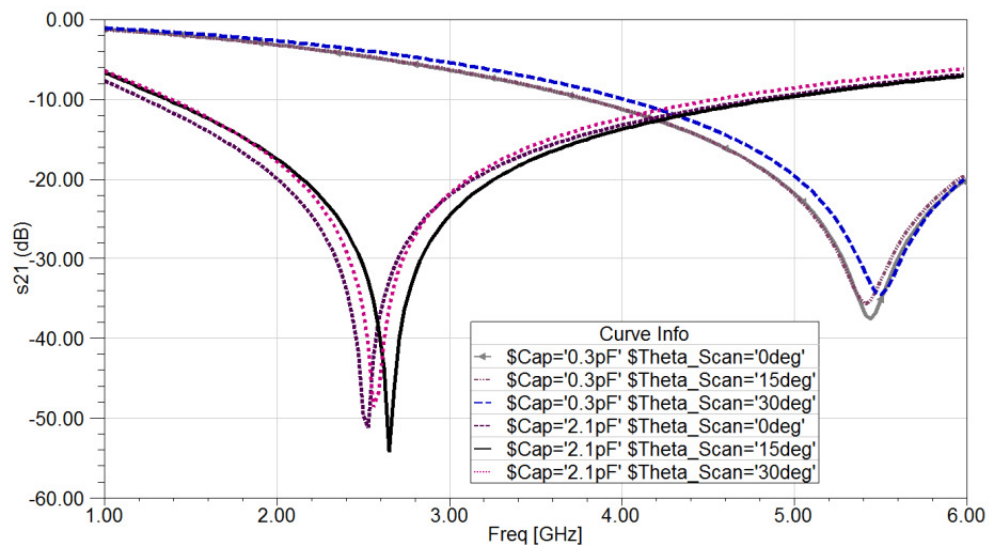

Figure 7. $\mathrm{S}_{21}$ curves for different incidence angles (TM polarization, $\theta=0^{0}, \theta=15^{0}, \theta=30^{0}$ ) 
Electrical and Electronics Engineering: An International Journal (ELELIJ) Vol.6, No.3, August 2017

\section{Conclusions}

Band stop FSS geometry with having wide tuning feature is proposed in this work. Minimum $30 \mathrm{~dB}$ attenuation is achieved in the desired frequency band between $2.42 \mathrm{GHz}$ and $5.96 \mathrm{GHz}$ frequencies. By adding capacitive edges to the end of legs of "Four Legged Loaded" FSS geometry frequency tuning range is almost increased $\% 11$ by comparing with the "Four Legged Loaded" element geometry. Obtained thickness of the structure is only $0.8 \mathrm{~mm}$ which also gives the possibility of using this design as a structural surface material for blocking the ISM signals.

\section{ACKNOWLEDGEMENTS}

We would like to thank TUBITAK (project no: 115E225) and Istanbul Technical University BAP Foundation (project no: 37010) for funding this work.

\section{REFERENCES}

[1] B. A. Munk, Frequency Selective Surfaces - Theory and Design. New York: John Wiley and Sons. Inc., 2000.

[2] T.K.Wu, Frequency Selective Surface and Grid Array: Wiley Interscience Publication, 1995.

[3] B. Hooberman, "Everything you ever wanted to know about frequency-selective surface filters but were afraid to ask," calvin. phys. columbia. edu/groupweb/filter. pdf, 2005.

[4] R. Mittra, C. H. Chan, and T. Cwik, "Techniques for analyzing frequency selective surfaces-a review," Proceedings of the IEEE, vol. 76, pp. 1593-1615, 1988.

[5] B. A. Munk, Finite antenna arrays and FSS: John Wiley \& Sons, 2003.

[6] J. C. Vardaxoglou, Frequency selective surfaces: analysis and design: Research Studies Press, 1997.

[7] S. N. Azemi, K. Ghorbani, and W. S. Rowe, "A reconfigurable FSS using a spring resonator element," IEEE Antennas and Wireless Propagation Letters, vol. 12, pp. 781-784, 2013.

[8] L. Bao-Qin, Q. Shao-Bo, T. Chuang-Ming, Z. Hang, Z. Heng-Yang, and L. Wei, "Varactor-tunable frequency selective surface with an embedded bias network," Chinese Physics B, vol. 22, p. 094103, 2013.

[9] F. Bayatpur and K. Sarabandi, "Tuning Performance of Metamaterial-Based Frequency Selective Surfaces," IEEE Transactions on Antennas and Propagation, vol. 57, pp. 590-592, Feb 2009.

[10] T. Chang, R. J. Langley, and E. A. Parker, "Frequency selective surfaces on biased ferrite substrates," Electronics Letters, vol. 30, pp. 1193-1194, Jul 211994.

[11] K. Fuchi, T. Junyan, B. Crowgey, A. R. Diaz, E. J. Rothwell, and R. O. Ouedraogo, "Origami Tunable Frequency Selective Surfaces," IEEE Antennas and Wireless Propagation Letters, vol. 11, pp. 473-475, 2012.

[12] T. George, W. R. Buchwald, M. S. Islam, J. Hendrickson, J. W. Cleary, A. K. Dutta, et al., "Active frequency selective surfaces," vol. 8725, p. 87250A, 2013.

[13] M. Li and N. Behdad, "Fluidically tunable frequency selective/phase shifting surfaces for high-power microwave applications," IEEE Transactions on Antennas and Propagation, vol. 60, pp. 2748-2759, Jun 2012.

[14] M. Li, B. Yu, and N. Behdad, "Liquid-tunable frequency selective surfaces," IEEE Microwave and wireless components letters, vol. 20, pp. 423-425, Aug 2010.

[15] S. M. Mahmood and T. A. Denidni, "Switchable Square Loop Frequency Selective Surface," Progress In Electromagnetics Research Letters, vol. 57, pp. 61-64, 2015.

[16] B. Sanz-Izquierdo and E. A. Parker, "Dual Polarized Reconfigurable Frequency Selective Surfaces," IEEE Transactions on Antennas and Propagation, vol. 62, pp. 764-771, Feb 2014.

[17] B. Sanz-Izquierdo, E. A. Parker, J. B. Robertson, and J. C. Batchelor, "Tuning technique for active FSS arrays," Electronics Letters, vol. 45, p. 1107, Oct 222009.

[18] H. Zhou, X. Wang, S. Qu, L. Zheng, H. Yuan, M. Yan, et al., "Dual-polarized FSS with Wide Frequency Tunability and Simple Bias Network," Session 1P0, p. 352, 2014.

[19] L. Derek and K. Sowerby, "Shielding strategies for interference mitigation in indoor wireless communications with frequency selective surfaces," in Antennas and Propagation Society International Symposium, 2005 IEEE, Washington D.C., 2005, pp. 260,263. 
[20] M. Raspopoulos, F. A. Chaudhry, and S. Stavrou, "Radio propagation in frequency selective buildings," European Transactions on Telecommunications, vol. 17, pp. 407-413, May-Jun 2006.

[21] M. Raspopoulos and S. Stavrou, "Frequency Selective Buildings Through Frequency Selective Surfaces," IEEE Transactions on Antennas and Propagation, vol. 59, pp. 2998-3005, Aug 2011.

[22] L. Y. Seng, M. F. A. Malek, W. F. Hoon, L. W. Leong, N. Saudin, L. Mohamed, et al., "Frequency selective surface for enhance wlan applications," in Wireless Technology and Applications (ISWTA), 2012 IEEE Symposium on, 2012, pp. 81-84.

[23] G. H. H. Sung, K. W. Sowerby, M. J. Neve, and A. G. Williamson, "A frequency-selective wall for interference reduction in wireless indoor environments," Ieee Antennas and Propagation Magazine, vol. 48, pp. 29-37, Oct 2006.

[24] H.-H. Sung, "Frequency selective wallpaper for mitigating indoor wireless interference," ResearchSpace@ Auckland, 2006.

[25] I. Ullah, D. Habibi, Z. Xiaoli, and G. Kiani, "Design of RF/Microwave efficient buildings using frequency selective surface," 2011 Ieee 22nd International Symposium on Personal Indoor and Mobile Radio Communications (Pimrc), pp. 2070-2074, 2011.

[26] C. Mias and J. H. Yap, "A varactor-tunable high impedance surface with a resistive-lumped-element biasing grid," IEEE Transactions on Antennas and Propagation, vol. 55, pp. 1955-1962, Jul 2007.

[27] H. Zahra, S. Rafique, M. F. Shafique, and K. P. Esselle, "A switchable frequency selective surface based on a modified Jerusalem-cross unit cell," in Antennas and Propagation (EuCAP), 2015 9th European Conference on, 2015, pp. 1-2.

[28] L. Boccia, I. Russo, G. Amendola, and G. Di Massa, "Tunable frequency-selective surfaces for beamsteering applications," Electronics letters, vol. 45, p. 1, Nov 192009.

[29] M. Safari, C. Shafai, and L. Shafai, "X-Band Tunable Frequency Selective Surface Using MEMS Capacitive Loads," IEEE Transactions on Antennas and Propagation, vol. 63, pp. 1014-1021, Mar 2015.

\section{AUTHORS}

Bora Döken received the B.Sc. degree in electric and electronic engineering and M.Sc. degree from "Satellite Communication and Remote Sensing" department of Informatics Institute from the Istanbul Technical University, Istanbul, Turkey, in 1992 and 2010, respectively. He is currently working toward the Ph.D. degree at the Informatics Institute of Istanbul Technical University. After working in various positions in the private sector, he became an Instructor at the Istanbul Technical University from October 2007. He is currently involved in the areas of FSSs, periodic structures, antennas, and metamaterials.

Mesut Kartal (M'91) received his MS degree in 1993 and Ph.D. degree in 2000. Currently, he is an associate professor in the Istanbul Technical University, Department of Electronics and Communication Engineering. His research interests include remote sensing, inverse problems, RF and microwave design engineering, as well as modelling, design, simulations and analysis, and CAD techniques in high frequency region.

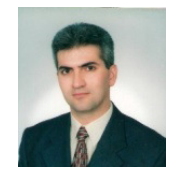


Electrical and Electronics Engineering: An International Journal (ELELIJ) Vol.6, No.3, August 2017

INTENTIONAL BLANK 